\title{
CORRECTION
}

\section{Correction to: Topology Optimization of 3D-printed joints under crash loads using Evolutionary Algorithms}

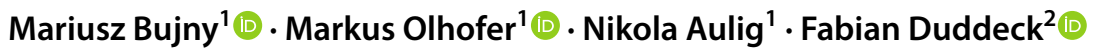

Published online: 18 October 2021

๑) Springer-Verlag GmbH Germany, part of Springer Nature 2021

\section{Correction to:}

Structural and Multidisciplinary Optimization https://doi.org/10.1007/s00158-021-03053-4

In the original publication of the article, Eqs. 12 to 14 were published incorrectly. The correct equations are provided in this correction. In Fig. 6b, the caption "Hybrid space frame structure by LZN, EDAG, BLM, and Concept Laser [74]" should be read as "Hybrid space frame structure by LZN, EDAG, BLM, and Concept Laser (LZN 2017)”.

$$
\begin{gathered}
x_{e_{b i}{ }^{\prime}}= \begin{cases}0, & \text { if } x_{e_{b i}}<0 \\
w_{\mathrm{dd}}, & \text { if } x_{e_{b i}}>w_{\mathrm{dd}} \\
x_{e_{b i}} & \text { otherwise, }\end{cases} \\
y_{e_{b i}{ }^{\prime}}= \begin{cases}0, & \text { if } y_{e_{b i}}<0 \\
h_{\mathrm{dd}}, & \text { if } y_{e_{b i}}>h_{\mathrm{dd}} \\
y_{e_{b i}} & \text { otherwise, }\end{cases} \\
z_{e_{b i}{ }^{\prime}}= \begin{cases}0, & \text { if } z_{e_{b i}}<0 \\
d_{\mathrm{dd}}, & \text { if } z_{e_{b i}}>d_{\mathrm{dd}} \\
z_{e_{b i}} & \text { otherwise, }\end{cases}
\end{gathered}
$$

Publisher's Note Springer Nature remains neutral with regard to jurisdictional claims in published maps and institutional affiliations.

The original article can be found online at https://doi.org/10.1007/ s00158-021-03053-4.

Mariusz Bujny

mariusz.bujny@honda-ri.de

1 Honda Research Institute Europe GmbH, Carl-Legien-Str. 30, 63073 Offenbach/Main, Germany

2 Faculty of Civil, Geo, and Environmental Engineering, Technische Universität München, Arcisstr. 21, 80333 Munich, Germany 